\title{
The Interplay of Shape and Crystalline Anisotropies in Plasmonic Semiconductor Nanocrystals
}

\author{
Jongwook Kim, ${ }^{*}{ }^{\dagger}$ Ankit Agrawal, ${ }^{\dagger}$ Franziska Krieg, ${ }^{\dagger, \dagger, \S}$ Amy Bergerud, ${ }^{\dagger, \|}$ and Delia J. Milliron*, ${ }^{\dagger}$ \\ ${ }^{\dagger}$ McKetta Department of Chemical Engineering, University of Texas at Austin, Austin, Texas 78712, United States \\ ${ }^{\ddagger}$ Institute of Inorganic Chemistry, Department of Chemistry and Applied Biosciences, ETH Zurich, Vladimir-Prelog-Weg 1, 8093 \\ Zurich, Switzerland \\ ${ }^{\S}$ Empa-Swiss Federal Laboratories for Materials Science and Technology, Uberlandstrasse 129, 8600, Dubendorf, Switzerland \\ "Department of Materials Science \& Engineering, University of California, Berkeley, Berkeley, California 94720, United States
}

\section{Supporting Information}

ABSTRACT: Doped semiconductor nanocrystals are an emerging class of materials hosting localized surface plasmon resonance (LSPR) over a wide optical range. Studies so far have focused on tuning LSPR frequency by controlling the dopant and carrier concentrations in diverse semiconductor materials. However, the influence of anisotropic nanocrystal shape and of intrinsic crystal structure on LSPR remain poorly explored. Here, we illustrate how these two factors collaborate to determine LSPR characteristics in hexagonal cesium-doped tungsten oxide nanocrystals. The effect of shape anisotropy is systematically analyzed via synthetic control of nanocrystal aspect ratio (AR), from disks to nanorods. We demonstrate the dominant influence of crystalline anisotropy, which

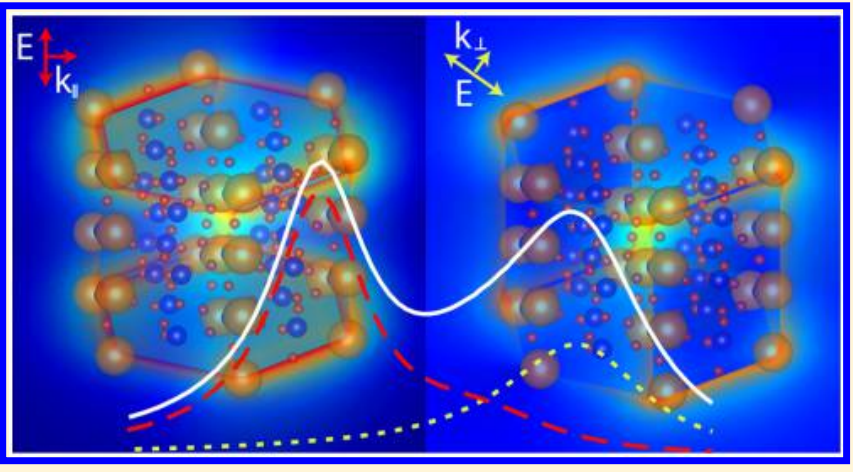
uniquely causes strong LSPR band-splitting into two distinct peaks with comparable intensities. Modeling typically used to rationalize particle shape effects is refined by taking into account the anisotropic dielectric function due to crystalline anisotropy, thus fully accounting for the AR-dependent evolution of multiband LSPR spectra. This new insight into LSPR of semiconductor nanocrystals provides a novel strategy for an exquisite tuning of LSPR line shape.

KEYWORDS: Doped semiconductor, tungsten bronze, nanorod, platelet, metal oxide

$\mathrm{L}$ ocalized surface plasmon resonance (LSPR) in metal nanoparticles or nanostructures has been extensively studied and utilized practically for sensing and manipulation of light. ${ }^{1-4}$ Gold and silver are typical LSPR host materials as their high free electron densities $\left(\sim 10^{23} / \mathrm{cm}^{3}\right)$ create resonances in the visible region. ${ }^{5}$ Employing an anisotropic particle shape allows for tuning of the LSPR, giving rise to variable resonance frequency $\left(\omega_{\text {lsp }}\right)$ via LSPR band-splitting that is inaccessible with (pseudo-) spherical particles. ${ }^{6}$ For instance, by changing the aspect ratio (AR) of gold nanorods, the longitudinal LSPR mode can be shifted to the near-infrared (NIR), while the transverse mode remains in the visible. ${ }^{7}$

In doped semiconductor nanocrystals, highly variable carrier density, $N\left(10^{18} \sim 10^{22} / \mathrm{cm}^{3}\right)$, enables $\omega_{\text {lsp }}$ over the entire infrared region. ${ }^{8-10}$ However, the connections between LSPR properties and materials composition and structure are still being established for this novel class of plasmonic materials. Especially as a broad range of doped semiconductor nanocrystal compositions (e.g., group IV elements, metal oxides, metal chalcogenides, and nitrides) are studied for plasmonic applications, ${ }^{9,10}$ there is a pressing need to rationalize and control the widely tunable LSPR in the infrared range. So far, the primary means to tune the $\omega_{\text {lsp }}$ of these nanocrystals has been to vary the doping level by controlling the concentration of vacancies (e.g., $\mathrm{Cu}_{2-x} \mathrm{~S}$, InN, $\left.\mathrm{WO}_{3-x}\right)^{8,11-15}$ or extrinsic dopants (e.g., $\mathrm{Sb}: \mathrm{SnO}_{2}, \mathrm{Sn}: \mathrm{InO}_{2}, \mathrm{Al}: \mathrm{ZnO}, \mathrm{P}$-doped $\mathrm{Si}$ ). ${ }^{16-19} \mathrm{~N}$ can thus be varied by 2 orders of magnitude, a tunability that has no parallel in classical metal plasmonics. Moreover, $N$ and therefore $\omega_{\text {lsp }}$ can be modulated postsynthetically by chem$\mathrm{ical}^{14}{ }^{14}$ electrochemical, ${ }^{20,21}$ or photoelectrochemical ${ }^{22,23}$ charging and discharging. Photoelectrochemical charging, or photodoping, in which photoexcited holes are scavenged to stabilize conduction band electrons, was used to compare the $\omega_{\text {lsp }}$ in $\mathrm{Sn}: \mathrm{In}_{2} \mathrm{O}_{3}$ with different $\mathrm{Sn}$ doping levels that had been postsynthetically adjusted to have comparable $N .^{23}$ The $\omega_{\text {lsp }}$ varied with $\mathrm{Sn}$ concentration, highlighting that composition impacts LSPR properties beyond the role of dopants in balancing the charge of free carriers.

While rapid progress has been made to understand how variable doping influences LSPR of semiconductor nanocryst-

\footnotetext{
Received: April 3, 2016

Revised: April 28, 2016

Published: May 16, 2016
} 

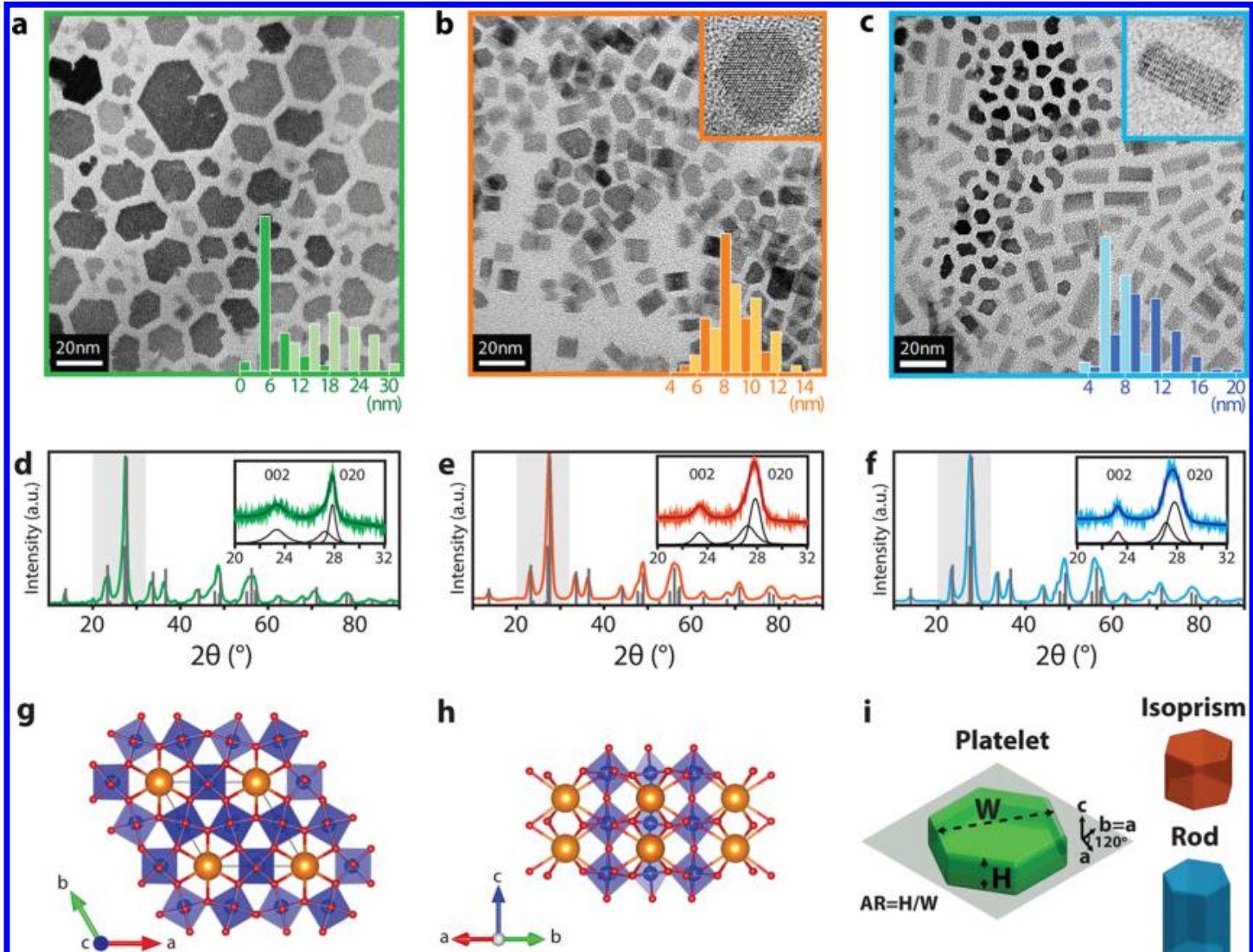

h

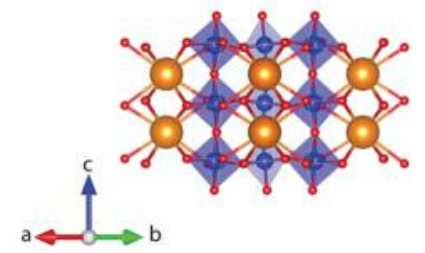

i

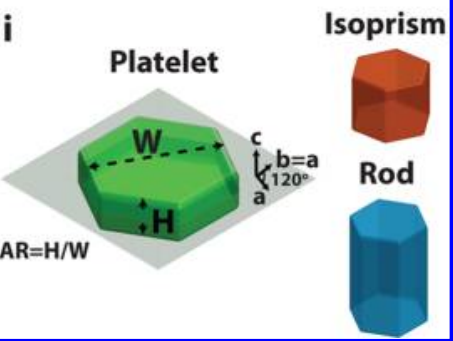

Figure 1. Hexagonal cesium-doped tungsten oxide (h-Cs: $\mathrm{WO}_{3}$ ) nanocrystals with different aspect ratios. (a-c) Transmission electron micrographs (TEM) of h-Cs: $\mathrm{WO}_{3}$ nanocrystals in the shape of (a) platelets, (b) iso-prisms, and (c) rods. The bottom right insets in a-c are size distribution histograms for the average height (dark color bars) and width (light color bars) of nanocrystals. Average sizes are listed in Table 1. (d-f) Powder X-ray diffraction (XRD) patterns for the h-Cs: $\mathrm{WO}_{3}$ nanocrystal samples corresponding to a-c. The insets in d-f are high-resolution XRD patterns of the gray regions, containing the (002) and (020) peaks that exhibit size-dependent broadening corresponding, respectively, to the height and width of nanocrystals. $(\mathrm{g}-\mathrm{h})$ Illustration of the $\mathrm{h}-\mathrm{Cs}: \mathrm{WO}_{3}$ lattice structure projection on the $(\mathrm{g})$ basal plane and $(\mathrm{h})$ prismatic plane: Cs (gold), $\mathrm{W}$ (blue), and $\mathrm{O}$ (red). (i) Schematic illustration of nanocrystal shape corresponding to a-c showing the conventions used in this paper.

als, the effect of anisotropic particle shape has been inconsistently reported and has only rarely been quantitatively rationalized. $^{24,25}$ As stated above, shape anisotropy is wellestablished as a dominant means of LSPR tuning in metal nanoparticles, which motivates investigation of shape effects in plasmonic semiconductor nanocrystals. Faceted octahedral In:CdO nanocrystals exhibited multiband LSPR that were well matched by simulated spectra in which deconvoluted dipolar spatial modes contributed to the line shape, ${ }^{25,26}$ whereas $\mathrm{WO}_{3-x}$ nanorods and $\mathrm{Cu}_{2-x} \mathrm{Te}$ platelets, rods, and tetrapods have not shown LSPR band-splitting despite their highly anisotropic morphologies. ${ }^{15,27}$ In the case of $\mathrm{Cu}_{2-x} \mathrm{~S}$ nanodisks, two widely separated peaks were observed and assigned to in-plane and out-of-plane modes, though the peak shifts with changing AR were unexpectedly small. ${ }^{28}$ Meanwhile, only one AR-dependent resonance peak was observed for covellite $\mathrm{CuS}$ nanoplatelets, which was assigned to the in-plane mode. ${ }^{24}$ To rationalize the literature on copper chalcogenide nanocrystal LSPR, the question of how the anisotropic (layered) crystal structure influences LSPR properties has been raised. ${ }^{27,28}$ After all, unlike classical plasmonic metals, which are typically isotropic, compound semiconductors commonly exhibit anisotropic crystal structures. Hence, we sought to understand how nanocrystal shape anisotropy and the underlying crystalline anisotropy might collaborate to produce the observed optical properties of these emerging plasmonic nanomaterials.

In this study, h-Cs: $\mathrm{WO}_{3}$ nanocrystals were investigated as a prototypical material since the hexagonal structure exhibits strong crystalline anisotropy. We modified our previously reported synthesis of h-Cs: $\mathrm{WO}_{3}$ nanocrystals ${ }^{29}$ to gain control over $\mathrm{AR}$, producing hexagonal prisms with $\mathrm{AR}$ from 0.3 to 2 . Figure $1 \mathrm{a}-\mathrm{c}$ displays the transmission electron microscopy (TEM) images for three representative samples, namely, platelets, iso-prisms, and rods with an $\mathrm{AR}$ of $0.32,0.87$, and 1.69 , respectively. XRD patterns (Figure $1 \mathrm{~d}-\mathrm{f}$ ) verify that they share the same hexagonal crystal phase (ICSD number 56223). $\mathrm{AR}$ is defined as the height $(H)$ of the prism divided by the inplane width $(W)$ parallel to the basal (002) plane (Figure 1gi). For each sample, $W, H$, and $\mathrm{AR}$ values of individual nanocrystals were assessed from multiple TEM images (Supplementary Figure S1). The histograms of $W$ and $H$ (insets in Figure 1a-c) clearly show the distinct morphology of each sample, despite the obvious size heterogeneity. ARs were also calculated via the Scherrer formula, ${ }^{30}$ with the $(002)$ and (020) XRD peaks exhibiting size-dependent broadening corresponding to $H$ and $W$, respectively, and found to be generally consistent with the results of TEM analysis (insets in Figure 1d-f, Table 1, and Table S1). The Scherrer-derived AR was employed in all subsequent analysis considering the 
Table 1. Nanocrystal Dimensions Obtained by Analyzing TEM Images and XRD Patterns

\begin{tabular}{lccccc} 
& \multicolumn{3}{c}{ size analysis by TEM } & & $\begin{array}{c}\text { Scherrer analysis } \\
\text { of XRD data }\end{array}$ \\
\cline { 2 - 4 } \cline { 5 - 6 } $\begin{array}{c}\text { nanocrystal } \\
\text { sample }\end{array}$ & $\begin{array}{c}\text { height }(H) \\
(\mathrm{nm})\end{array}$ & $\begin{array}{c}\text { width }(W) \\
(\mathrm{nm})\end{array}$ & $\begin{array}{c}\text { aspect ratio } \\
(\mathrm{AR})\end{array}$ & & $\begin{array}{c}\text { aspect ratio } \\
(\mathrm{AR})\end{array}$ \\
platelet & $7.0 \pm 3.0$ & $21.4 \pm 5.3$ & $0.46 \pm 0.17$ & & 0.32 \\
iso-prism & $9.8 \pm 1.8$ & $9.0 \pm 1.3$ & $0.94 \pm 0.17$ & & 0.87 \\
rod & $11.3 \pm 3.0$ & $5.5 \pm 1.5$ & $2.10 \pm 0.44$ & & 1.69 \\
\hline
\end{tabular}

possible statistical errors from the limited number of nanocrystals measured by TEM.

The optical absorption spectra of the three representative colloidal samples vary systematically with AR (Figure 2a). The platelet spectrum $(\mathrm{AR}=0.32)$ contains two peaks-a highfrequency peak $\left(\omega_{\text {lsp1 } 1}\right)$ at $14140 \mathrm{~cm}^{-1}$ and a low-frequency peak $\left(\omega_{\text {lsp2 } 2}\right)$ at $6480 \mathrm{~cm}^{-1}$. The resulting peak separation $\left(\Delta \omega_{\text {lsp }}\right)$ of $7660 \mathrm{~cm}^{-1}$ is quite large. As AR increases, $\omega_{\text {lspl }}$ redshifts, while $\omega_{\text {lsp2 }}$ blueshifts, resulting in a decrease in $\Delta \omega_{\text {lsp }}$ (orange and blue lines, green circles in Figure $2 \mathrm{~b}$ ). According to the established model for LSPR band-splitting developed in the context of classical metal nanoparticles, ${ }^{31} \omega_{\mathrm{lsp} 1}$ of the platelets is expected to be the longitudinal LSPR (out-of-plane) mode and $\omega_{\text {lsp2 }}$ is to be the transverse (in-plane) mode. Assuming dielectric isotropy, as in conventional plasmonic metals, the two peaks are expected to coincide $\left(\Delta \omega_{\text {lsp }}=0\right)$ when $\mathrm{AR} \approx 1$, and to crossover and separate once again when AR > 1 (blue stars in Figure 2b). Instead, we observe a striking deviation from this expectation, with $\Delta \omega_{\text {lsp }}$ continuously decreasing, without crossover, even as the AR increases to well over 1 (Figure 2a, green circles in Figure $2 b$ ). This result suggests that intrinsic crystalline anisotropy may strongly modify the expectations for LSPR of anisotropically shaped nanocrystals, even qualitatively changing the interpretation and assignment of multipeaked spectra. According to the Drude model,

$$
\vec{\epsilon}_{\mathrm{D}}(\omega)=\vec{\epsilon}_{\infty}(\omega)-\frac{\vec{\omega}_{\mathrm{p}}^{2}}{\omega^{2}-\omega \vec{\gamma}} \quad \vec{\omega}_{\mathrm{p}}=\sqrt{\frac{N e^{2}}{\epsilon_{0} \vec{m}_{\mathrm{e}}}}
$$

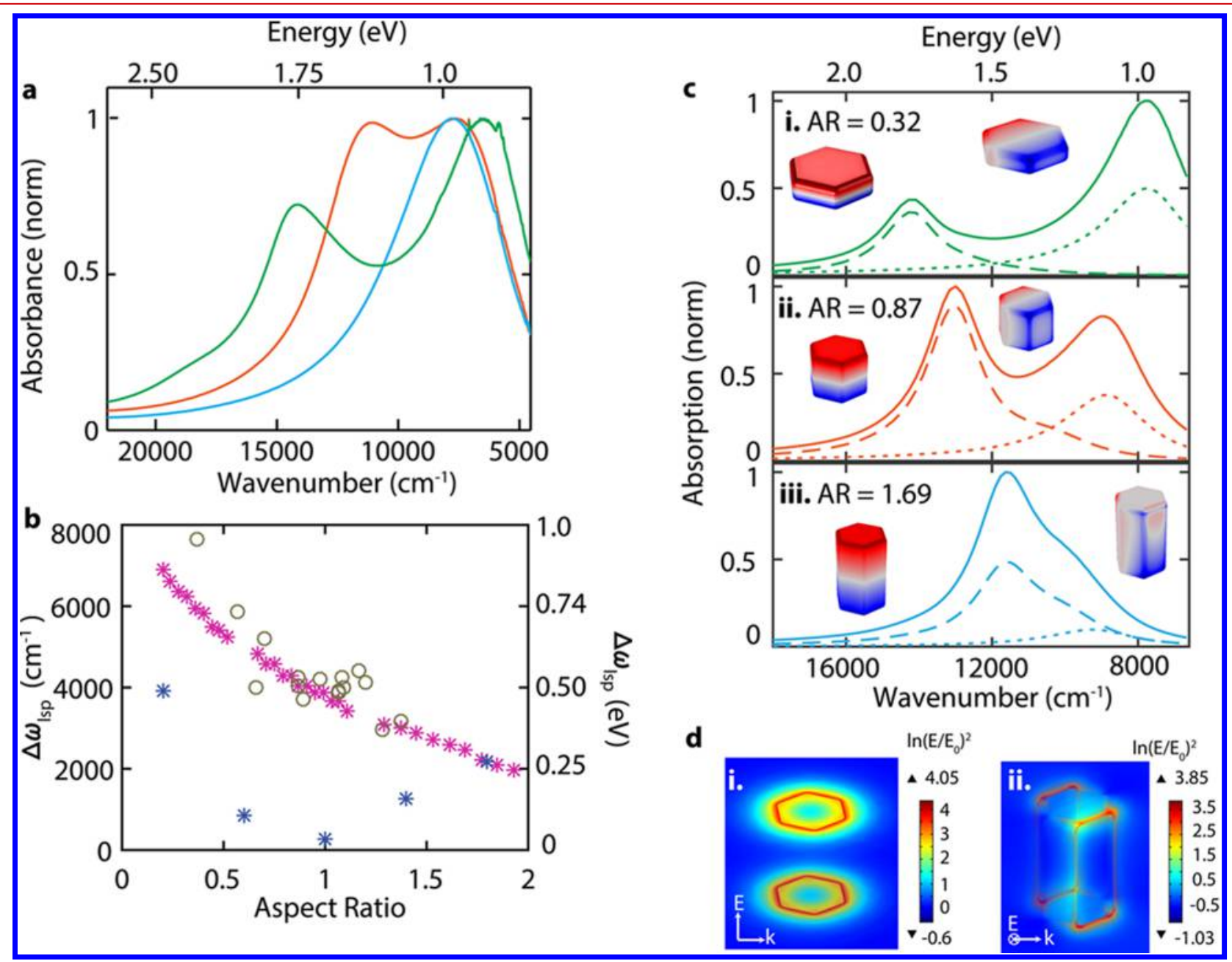

Figure 2. LSPR of h-Cs: $\mathbf{W O}_{3}$ nanocrystals. (a) Experimental optical absorption spectra of h-Cs: $\mathrm{WO}_{3}$ nanocrystal samples of platelets (green), isoprisms (orange), and rods (blue) dispersed in tetrachloroethylene showing the aspect ratio-dependence of LSPR. (b) Plot of LSPR peak splitting $\left(\Delta \omega_{\text {lsp }}\right)$ vs particle aspect ratio obtained experimentally with synthesized nanocrystals (open circles) and by theoretical modeling assuming isotropic (blue stars) or anisotropic (pink stars) dielectric functions. (c) Theoretically simulated LSPR spectra for platelet-, iso-prism-, and rod-shaped nanocrystals (top to bottom) using the anisotropic dielectric function of $\mathrm{h}-\mathrm{Cs}: \mathrm{WO}_{3}$. Solid lines show the overall absorption profiles, summing one longitudinal mode (dashed lines) and two identical transverse modes (dotted lines). The insets show the simulated dipolar surface charge distributions at each peak frequency of the longitudinal and transverse modes. (d) Color map of the simulated near-field enhancement for a rod excited at the two LSPR peak frequencies. 


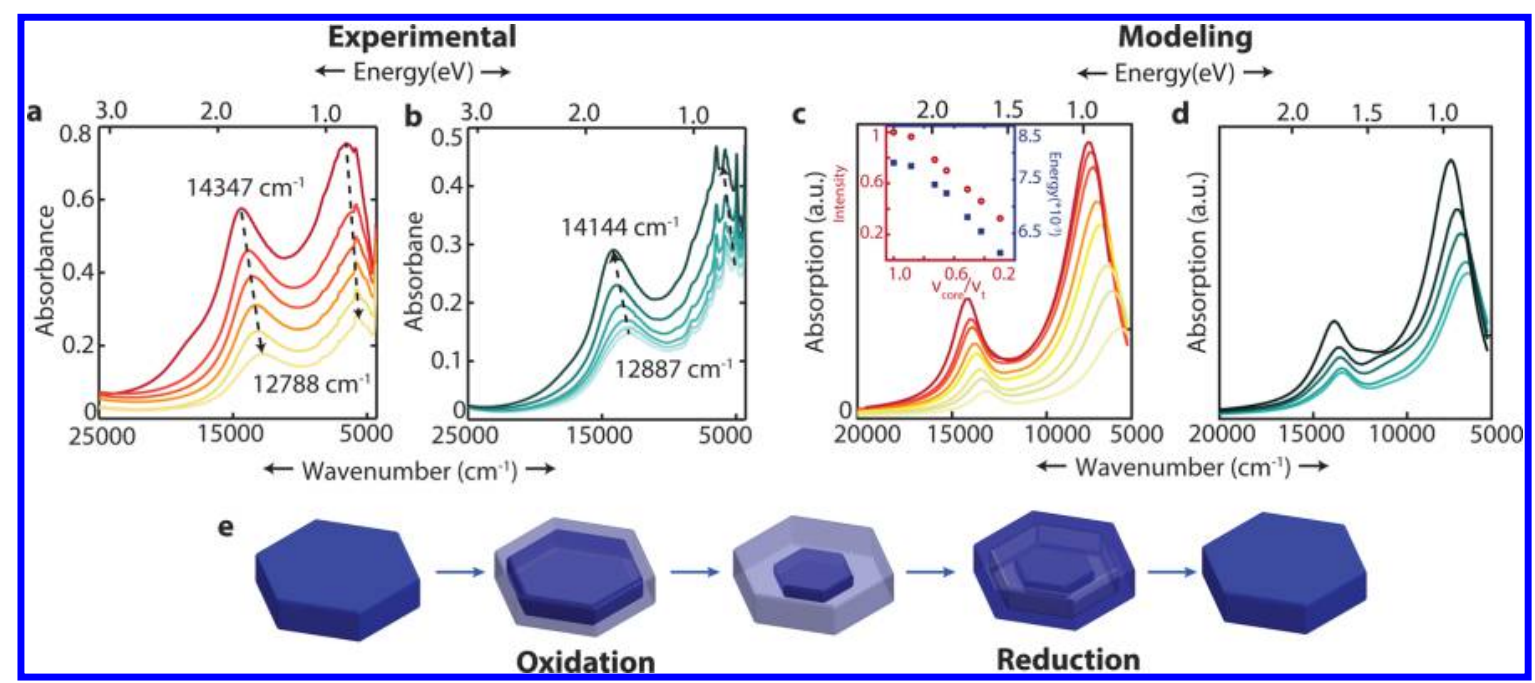

Figure 3. Redox-mediated modulation of LSPR. (a-b) Dynamic optical absorption spectra of h-Cs:WO $\mathrm{W}_{3}$ platelets undergoing (a) oxidation by exposure to air for up to $24 \mathrm{~h}$ and (b) reduction by incremental addition of hydrazine. (c-d) Simulated evolution of LSPR for (c) oxidation and (d) reduction. (e) The simulation was performed assuming oxidation proceeds by the growth of a fully oxidized shell with unchanged carrier density in the core, and reduction by the growth of fully reduced shell upon the previously oxidized volume. The inset in $\mathrm{c}$ is a plot of the longitudinal mode peak intensity (red dots) and peak position (black squares) versus unoxidized core volume during simulated oxidation, both showing an approximately linear relationship.

dielectric permittivity $\left(\vec{\epsilon}_{\mathrm{D}}\right)$ and bulk plasma frequency $\left(\vec{\omega}_{\mathrm{p}}\right)$ are determined by free carrier concentration $(N)$, elementary electronic charge $(e)$, the permittivity of free space $\left(\varepsilon_{0}\right)$, highfrequency permittivity $\left(\vec{\epsilon}_{\infty}\right)$, damping $(\vec{\gamma})$, and electron effective mass $\left(\vec{m}_{\mathrm{e}}\right)$, the last three of which can vary with direction in crystals with anisotropic structures. In case of bulk h-Cs: $\mathrm{WO}_{3}$, density functional theory calculations ${ }^{32}$ and reflection spectroscopic measurements ${ }^{33}$ have shown that $\vec{m}_{\mathrm{e}}$, $\vec{\epsilon}_{\infty}$, and $\vec{\gamma}$ are all strongly dependent upon the lattice direction. In particular, the significantly lower $m_{\mathrm{e}}$ along the $c$-axis compared to that parallel to the basal plane results in the longitudinal $\omega_{\mathrm{p}}$ being 1.5 times higher than the transverse $\omega_{\mathrm{p}}$. LSPR of h-Cs: $\mathrm{WO}_{3}$ nanocrystals should accordingly exhibit a similar anisotropy in $\omega_{\text {lsp }}$ in addition to the effect of shape anisotropy. The unexpected observation of a substantial peak splitting $\left(\Delta \omega_{\text {lsp }}=4000 \mathrm{~cm}^{-1}\right)$ when $\mathrm{AR} \approx 1$ (Figure $\left.2 \mathrm{~b}\right)$ is thus a natural consequence of the strong crystalline anisotropy of $\mathrm{h}$ Cs: $\mathrm{WO}_{3}$. The revised calculation of $\Delta \omega_{\text {lsp }}$ vs AR, employing an anisotropic $\vec{\omega}_{\mathrm{p}}$ (pink stars in Figure $2 \mathrm{~b}$ ), matches well with the experimental data (green circles in Figure $2 \mathrm{~b}$ ).

In order to theoretically investigate the implications of such an interplay of shape and crystalline anisotropies, electromagnetic field simulations were performed by setting anisotropic dielectric functions along different lattice directions (see the SI for computational method details) and varying the AR of the nanocrystal (Figure S3). In the simulated LSPR spectra (Figure 2c) the longitudinal and transverse modes are visibly separated when $A R=0.32$ and 0.87 and overlay only when $\mathrm{AR}=1.69$, which is in good agreement with the experimental data (Figure 2a). The deconvoluted spectra showing each LSPR mode (dashed and dotted lines in Figure $2 c$ ) and the surface charge distribution profiles reveal that each peak corresponds to a distinct dipolar mode (Figure 2c, insets and Figure S5). By contrast, simulated spectra for an isotropic dielectric function (Figure S4) exhibit two modes that overlap over the entire range of AR (from 0.2 to 1.8 ) due to smaller
$\Delta \omega_{\text {lsp }}$ and that have strongly divergent peak intensities at low or high AR, yielding a single strong peak and a shoulder. These results confirm that crystalline anisotropy is a major contributor to the LSPR band-splitting and overall line shape in h-Cs: $\mathrm{WO}_{3}$.

Furthermore, the near-field intensity maps (Figure $2 \mathrm{~d}$ and S6) illustrate that up to 400 -fold enhancement is achievable for both longitudinal and transverse modes in a given anisotropic $\mathrm{h}-\mathrm{Cs}: \mathrm{WO}_{3}$ nanocrystal. This is a unique attribute of anisotropic crystalline hosts as isotropic hosts inevitably give rise to unbalanced field enhancement for the different modes. This balanced dual-mode LSPR property enables effective concentration of infrared light over a broad energy range and through a substantial region of space spanning all solid angles around the nanocrystal.

One can recognize that the experimental spectra (Figure 2a) are broader than the simulated ones (Figure $2 c$ ) and that the relative peak intensities are not precisely reproduced. A possible reason for these discrepancies is the polydispersity in the size and shape of nanocrystals that is obvious from TEM images (Figure $1 \mathrm{a}-\mathrm{c})$. Simulation parameters were thus adjusted to examine the validity of our model to describe these nonideal ensembles of nanocrystals. First, a Gaussian average of the nanocrystal AR (with fixed volume) was applied using the measured standard deviations from TEM (Table 1), resulting in broadened LSPR spectra for all three shapes (platelets, isoprisms, rods; Figure S7). It was also found that the longitudinal mode, corresponding to the lower $m_{\mathrm{e}}$, varies nonlinearly and more rapidly with changing $\mathrm{AR}$ in both intensity and peak position compared to the transverse mode, resulting in a higher relative intensity at the longitudinal peak (Figure S7). Second, the experimentally realized morphologies exhibit substantial "bites" taken out of the lateral facets, especially in the platelets. This morphology was mimicked by simulating the LSPR spectrum of a distinctively shaped platelet. Compared to the pristine shape, there was no noticeable change in the peak positions while a broadening of the transverse mode peak was observed (Figure S8). Therefore, the minor differences between experimental and simulated spectra can be accounted 
for by size and shape heterogeneity within each nanocrystal sample.

Considering that post-synthetic modulation of LSPR is a key feature of plasmonic semiconductor nanocrystals, we sought to understand how shape and crystalline anisotropy influence the spectral evolution of h-Cs: $\mathrm{WO}_{3}$ nanocrystals subjected to oxidation and reduction. It is well-known that free carriers in vacancy doped tungsten oxides are highly sensitive to oxygen partial pressure, where high levels of atmospheric oxygen can drive the insertion of oxygen, thus diminishing the free electron population. ${ }^{34}$ This effect, usually limited to the near-surface regime in bulk materials, has been shown to overwhelm the entire volume of small $\mathrm{WO}_{3}$ nanocrystals, and therefore the LSPR may be greatly diminished in intensity in oxidative conditions. ${ }^{15,35}$ We tracked the oxidation-induced quenching and redshift of LSPR in h-Cs: $\mathrm{WO}_{3}$ nanocrystals as a function of air exposure (Figure 3a). Moreover, by addition of hydrazine as a reducing agent, the oxidized nanocrystals could be reduced again, recovering the original line shape and peak positions (Figure $3 b$ ). The correlated response of the two peaks supports our description of the spectra as two distinct LSPR modes originating from the same free carriers; by contrast, if the two peaks reflected distinct electronic states they would have differing responses to the oxidation and reduction processes. The moderate peak shifts, while the peak intensities are strongly modulated, are inconsistent with a homogeneous depletion of $N$ (Figure S9a), but can be reproduced instead by assuming that oxidation proceeds from the surface. This was modeled by creating an oxidized, fully depleted shell and a shrinking core with unchanged carrier density, as depicted in Figure 3e. Similarly, the reduction process was modeled with a reduced shell growing progressively inward from the surface (Figure 3e). The simulated evolution of the LSPR spectra based on this redox mechanism (Figure $3 c-d$ ), in which peaks experience a moderate linear shift due to the dielectric environment imposed by the growing shell (Figure 3c, inset), is highly consistent with the experimental observations (Figure $3 a-b)$. Although the redox-mediated LSPR evolution in semiconductor nanocrystals has typically been described as a homogeneous modulation of $N,^{12,31}$ our results suggest this model should be revisited to ensure a realistic view of the electronic changes within the nanocrystals that underlie observed LSPR modulation.

To conclude, colloidally synthesized hexagonal phase Cs: $\mathrm{WO}_{3}$ nanocrystals exhibit strong aspect ratio-dependent LSPR absorption peaks that can only be explained via a cooperative influence of crystalline and shape anisotropies. This finding highlights the limitations of conventional treatments of LSPR that assume isotropic dielectric constants and attribute multimodal peaks uniquely to shape anisotropy effects. This understanding extends our ability to rationally tune LSPR line shape and near-field enhancement via synthetic control of shape and crystalline anisotropies of semiconductor nanocrystals. Such control may prove useful by enhancing light-matter interactions across a broad spectral and spatial region, leading to the advances in developing switchable optical devices, sensors, and waveguides. In particular, the demonstrated multimodal LSPR with near-equal intensities of h-Cs: $\mathrm{WO}_{3}$ nanocrystals covers the near-infrared (NIR) region of great importance in photonic, solar, and clinical applications while maintaining high visible transparency due to its wide band gap. Such an optical performance with the additional functionality of dynamic, reversible modulability is promising for sensing applications $^{36}$ and for energy saving NIR-selective electrochromic windows, ${ }^{35,37}$ which also motivates the additional study of the optical response of this doubly anisotropic nanomaterial to chemical or electrochemical stimuli.

\section{ASSOCIATED CONTENT}

\section{S Supporting Information}

The Supporting Information is available free of charge on the ACS Publications website at DOI: 10.1021/acs.nanolett.6b01390.

TEM, Scherrer analysis, solvent refractive index dependence of LSPR, and detailed modeling results (PDF)

\section{AUTHOR INFORMATION}

\section{Corresponding Authors}

*E-mail (J.K.): jong-wook.kim@polytechnique.edu.

*E-mail: (D.J.M.): milliron@che.utexas.edu.

\section{Author Contributions}

J.K., A.A., and F.K. contributed equally to this work. F.K. synthesized the materials, J.K. performed materials characterization and analysis, A.A. performed simulations, A.B. performed analysis of X-ray diffraction, D.J.M. and J.K. provided overall guidance, and J.K., A.A., and D.J.M. wrote the manuscript with critical input by all the authors.

\section{Notes}

The authors declare no competing financial interest.

\section{ACKNOWLEDGMENTS}

This research was supported by a U.S. Department of Energy (DOE) ARPA-E grant (J.K.) and a DOE SBIR grant (A.A.). A.B. was supported in part by a National Science Foundation Graduate Research Fellowship. D.J.M. acknowledges support by the Welch Foundation (F-1848).

\section{REFERENCES}

(1) Barnes, W. L.; Dereux, A.; Ebbesen, T. W. Surface plasmon subwavelength optics. Nature 2003, 424, 824-830.

(2) Maier, S. A.; Atwater, H. A. Plasmonics: Localization and guiding of electromagnetic energy in metal/dielectric structures. J. Appl. Phys. 2005, 98, 011101.

(3) Anker, J. N.; et al. Biosensing with plasmonic nanosensors. Nat. Mater. 2008, 7, 442-453.

(4) Linic, S.; Christopher, P.; Ingram, D. B. Plasmonic-metal nanostructures for efficient conversion of solar to chemical energy. Nat. Mater. 2011, 10, 911-921.

(5) Alvarez, M. M.; et al. Optical absorption spectra of nanocrystal gold molecules. J. Phys. Chem. B 1997, 101, 3706-3712.

(6) Kelly, K. L.; Coronado, E.; Zhao, L. L.; Schatz, G. C. The optical properties of metal nanoparticles: The influence of size, shape, and dielectric environment. J. Phys. Chem. B 2003, 107, 668-677.

(7) Pérez-Juste, J.; Pastoriza-Santos, I.; Liz-Marzán, L. M.; Mulvaney, P. Gold nanorods: Synthesis, characterization and applications. Coord. Chem. Rev. 2005, 249, 1870-1901.

(8) Luther, J. M.; Jain, P. K.; Ewers, T.; Alivisatos, A. P. Localized surface plasmon resonances arising from free carriers in doped quantum dots. Nat. Mater. 2011, 10, 361-366.

(9) Comin, A.; Manna, L. New materials for tunable plasmonic colloidal nanocrystals. Chem. Soc. Rev. 2014, 43, 3957-3975.

(10) Lounis, S. D.; Runnerstrom, E. L.; Llordés, A.; Milliron, D. J. Defect chemistry and plasmon physics of colloidal metal oxide nanocrystals. J. Phys. Chem. Lett. 2014, 5, 1564-1574.

(11) Hsu, S.-W.; Bryks, W.; Tao, A. R. Effects of carrier density and shape on the localized surface plasmon resonances of $\mathrm{Cu}_{2-x} \mathrm{~S}$ nanodisks. Chem. Mater. 2012, 24, 3765-3771. 
(12) Dorfs, D.; et al. Reversible tunability of the near-infrared valence band plasmon resonance in $\mathrm{Cu}_{2-x} \mathrm{Se}$ nanocrystals. J. Am. Chem. Soc. 2011, 133, 11175-11180.

(13) Kriegel, I.; et al. Tuning the excitonic and plasmonic properties of copper chalcogenide nanocrystals. J. Am. Chem. Soc. 2012, 134, $1583-1590$.

(14) Palomaki, P. K. B.; Miller, E. M.; Neale, N. R. Control of plasmonic and interband transitions in colloidal indium nitride nanocrystals. J. Am. Chem. Soc. 2013, 135, 14142-14150.

(15) Manthiram, K.; Alivisatos, A. P. Tunable localized surface plasmon resonances in tungsten oxide nanocrystals. J. Am. Chem. Soc. 2012, 134, 3995-3998.

(16) Nütz, T.; Felde, U. z.; Haase, M. Wet-chemical synthesis of doped nanoparticles: Blue-colored colloids of n-doped $\mathrm{SnO}_{2}: \mathrm{Sb}$. J. Chem. Phys. 1999, 110, 12142-12150.

(17) Kanehara, M.; Koike, H.; Yoshinaga, T.; Teranishi, T. Indium tin oxide nanoparticles with compositionally tunable surface plasmon resonance frequencies in the near-IR region. J. Am. Chem. Soc. 2009, 131, 17736-17737.

(18) Buonsanti, R.; Llordes, A.; Aloni, S.; Helms, B. A.; Milliron, D. J. Tunable infrared absorption and visible transparency of colloidal aluminum-doped zinc oxide nanocrystals. Nano Lett. 2011, 11, 47064710.

(19) Rowe, D. J.; Jeong, J. S.; Mkhoyan, K. A.; Kortshagen, U. R. Phosphorus-doped silicon nanocrystals exhibiting mid-infrared localized surface plasmon resonance. Nano Lett. 2013, 13, 1317-1322.

(20) zum Felde, U.; Haase, M.; Weller, H. Electrochromism of highly doped nanocrystalline $\mathrm{SnO}_{2}$ :Sb. J. Phys. Chem. B 2000, 104, 93889395.

(21) Garcia, G.; et al. Dynamically modulating the surface plasmon resonance of doped semiconductor nanocrystals. Nano Lett. 2011, 11, $4415-4420$.

(22) Schimpf, A. M.; Thakkar, N.; Gunthardt, C. E.; Masiello, D. J.; Gamelin, D. R. Charge-tunable quantum plasmons in colloidal semiconductor nanocrystals. ACS Nano 2014, 8, 1065-1072.

(23) Schimpf, A. M.; Lounis, S. D.; Runnerstrom, E. L.; Milliron, D. J.; Gamelin, D. R. Redox chemistries and plasmon energies of photodoped $\mathrm{In}_{2} \mathrm{O}_{3}$ and Sn-doped $\mathrm{In}_{2} \mathrm{O}_{3}$ (ITO) nanocrystals. J. Am. Chem. Soc. 2015, 137, 518-524.

(24) Liu, M.; et al. Room-temperature synthesis of covellite nanoplatelets with broadly tunable localized surface plasmon resonance. Chem. Mater. 2015, 27, 2584-2590.

(25) Agrawal, A.; Kriegel, I.; Milliron, D. J. Shape-dependent field enhancement and plasmon resonance of oxide nanocrystals. J. Phys. Chem. C 2015, 119, 6227-6238.

(26) Gordon, T. R.; et al. Shape-dependent plasmonic response and directed self-assembly in a new semiconductor building block, indiumdoped cadmium oxide (ICO). Nano Lett. 2013, 13, 2857-2863.

(27) Kriegel, I.; et al. Shedding light on vacancy-doped copper chalcogenides: shape-controlled synthesis, optical properties, and modeling of copper telluride nanocrystals with near-infrared plasmon resonances. ACS Nano 2013, 7, 4367-4377.

(28) Hsu, S.-W.; On, K.; Tao, A. R. Localized surface plasmon resonances of anisotropic semiconductor nanocrystals. J. Am. Chem. Soc. 2011, 133, 19072-19075.

(29) Mattox, T. M.; Bergerud, A.; Agrawal, A.; Milliron, D. J. Influence of shape on the surface plasmon resonance of tungsten bronze nanocrystals. Chem. Mater. 2014, 26, 1779-1784.

(30) Manna, L.; Scher, E. C.; Alivisatos, A. P. Synthesis of soluble and processable rod-, arrow-, teardrop-, and tetrapod-shaped CdSe nanocrystals. J. Am. Chem. Soc. 2000, 122, 12700-12706.

(31) Noguez, C. Surface Plasmons on Metal Nanoparticles: The influence of shape and physical environment. J. Phys. Chem. C 2007, 111, 3806-3819.

(32) Ingham, B.; Hendy, S. C.; Chong, S. V.; Tallon, J. L. Densityfunctional studies of tungsten trioxide, tungsten bronzes, and related systems. Phys. Rev. B: Condens. Matter Mater. Phys. 2005, 72, 075109.
(33) Hussain, A.; Gruehn, R.; Rüscher, C. H. Crystal growth of alkali metal tungsten bronzes $\mathrm{M}_{\mathrm{x}} \mathrm{WO}_{3}(\mathrm{M}=\mathrm{K}, \mathrm{Rb}, \mathrm{Cs})$, and their optical properties. J. Alloys Compd. 1997, 246, 51-61.

(34) Xin, Y.; et al. The optical properties of low infrared transmittance $\mathrm{WO}_{3-\mathrm{x}}$ nanocrystal thin films prepared by DC magnetron sputtering under different oxygen ratios. RSC Adv. 2015, 5, 57757-57763.

(35) Kim, J.; et al. Nanocomposite architecture for rapid, spectrallyselective electrochromic modulation of solar transmittance. Nano Lett. 2015, 15, 5574-5579.

(36) Mendelsberg, R. J.; et al. Dispersible plasmonic doped metal oxide nanocrystal sensors that optically track redox reactions in aqueous media with single-electron Sensitivity. Adv. Opt. Mater. 2015, 3, 1293-1300.

(37) Llordes, A.; Garcia, G.; Gazquez, J.; Milliron, D. J. Tunable nearinfrared and visible-light transmittance in nanocrystal-in-glass composites. Nature 2013, 500, 323-326. 